\title{
BOUNDED SETS IN INDUCTIVE LIMITS
}

\section{J. KUCERA AND K. MCKENNON}

\begin{abstract}
The Dieudonne-Schwartz theorem for bounded sets in strict inductive limits does not hold for general inductive limits. A set $B$ bounded in an inductive limit $E=$ ind $\lim E_{n}$ of locally convex spaces may not be contained in any $E_{n}$. If, however, each $E_{n}$ is closed in $E$, then $B$ is contained in some $E_{n}$, but may not be bounded there.
\end{abstract}

Let $E_{1} \subset E_{2} \subset \ldots$ be a sequence of locally convex spaces and $E=$ ind $\lim E_{n}$ their inductive limit (with respect to the identity maps id: $E_{n} \rightarrow$ $\left.E_{n+1}\right)$. It is proved in [2, Chapter 2, §12], that a set $B \subset E$ is bounded iff it is contained and bounded in some $E_{n}$, provided that

(H-1) $E_{n}$ is closed in $E_{n+1}$ for each $n \in N$,

(H-2) for each $n \in N$, the topology of $E_{n}$ coincides with the topology induced on $E_{n}$ by $E_{n+1}$.

These two hypotheses imply [2, Chapter 2, §12]

(H-3) $E_{n}$ is closed in $E$ for each $n \in N$.

If we replace $\mathrm{H}-1$ and $\mathrm{H}-2$ by $\mathrm{H}-3$, any set bounded in $E$ must still be contained in some $E_{n}$. But, as Example 1 shows, it may not be bounded in any $E_{m}, m \geqslant n$. Example 2 shows that, if we assume only $\mathrm{H}-1$ instead of $\mathrm{H}-3$, there may exist sets bounded in $E$ but not contained in any $E_{n}$.

TheOREM. Let $\mathrm{H}-3$ hold and $B$ be bounded in $E$. Then $B \subset E_{n}$ for some $n$.

Proof. Assume the contrary. Without loss of generality, we may assume that there exists a sequence $b_{1}, b_{2}, \ldots$ in $B$ such that $b_{n} \in E_{n} \backslash E_{n-1}$, $E_{0}=\{0\}$, for all $n \in N$.

Since $b_{1} \neq 0$, there exists a convex neighborhood $G_{1}$ of 0 in $E$ such that $b_{1} \notin G_{1}+G_{1}$. Put $V_{1}=G_{1} \cap E_{1}$. Then $V_{1}$ is a neighborhood of 0 in $E_{1}$ and $b_{1} \notin V_{1}$. Suppose that in each $E_{k}, k=1,2, \ldots, n$ a neighborhood $V_{k}$ of 0 was chosen so that $V_{1} \subset V_{2} \subset \cdots \subset V_{n}$ and $b_{m} / m \notin\left(\overline{V_{1}+\cdots+V_{n}}\right)$, $m=1,2, \ldots, n$. Put, for brevity, $W_{k}=\left(\overline{V_{1}+\cdots+V_{k}}\right)^{E}$. Since $E_{n}$ is closed in $E, W_{n} \subset E_{n}$ and there exists a convex neighborhood $G_{n+1}$ of 0 in $E$ such that

$$
\frac{1}{m} b_{m} \notin W_{n}+G_{n+1}+G_{n+1} \text { for all } m=1,2, \ldots, n+1 .
$$

Received by the editors August 25, 1977.

AMS (MOS) subject classifications (1970). Primary 46A05.

Key words and phrases. Locally convex space, inductive limit, bounded set, finest locally convex topology. 
The set $V_{n+1}=G_{n+1} \cap E_{n+1}$ is a neighborhood of 0 in $E_{n+1}$ and

$$
\begin{aligned}
W_{n+1}= & \left(\overline{V_{1}+\cdots+V_{n+1}}\right)^{E} \subset V_{1}+\cdots+V_{n+1} \\
& +G_{n+1} \subset V_{1}+\cdots+V_{n}+G_{n+1}+G_{n+1} \subset W_{n}+G_{n+1}+G_{n+1} .
\end{aligned}
$$

This implies $b_{m} / m \notin W_{n+1}$ for $m=1,2, \ldots, n+1$.

The union of the nest $\left\{W_{n} ; n \in N\right\}$ is a neighborhood of 0 in $E$ which does not contain any $b_{m} / m, m \in N$, and therefore does not absorb $B$.

The following notation is useful for our examples. For each $n \in N, n$ will denote the set $\{1,2, \ldots, n\}$ and $N \backslash n$ the complement of $n$ in $N$. Then, for instance, $A^{n} \times B^{N \backslash n}$ denotes the set of all sequences of which the first $n$ terms are in $A$ and the remainder in $B$.

EXAMPLE 1. Let $X$ be an infinite dimensional Banach space, $L$ its underlying linear space, and $Y$ the space $L$ endowed with its finest locally convex topology. Let, for each $n \in N, E_{n}$ be the locally convex product $X^{\underline{n}} \times Y^{N \backslash \underline{n}}$. As linear spaces, all the $E_{n}$ can be identified with $L^{N}$. The natural inductive $\operatorname{limit} E=$ ind $\lim E_{n}$ can be as well. That $\mathrm{H}-3$ holds, is trivial.

Claim. $E=X^{N}$. That the topology on $E$ is as fine as the product topology is evident. Let $W$ be any convex neighborhood of 0 in $E$. Then $W$ is a neighborhood of 0 in $E_{1}$ and so there exists some $n \in N$ and $U$ a neighborhood of 0 in $Y$ such that

$$
Q_{1} \equiv U^{n} \times L^{N \backslash \underline{n}} \subset W
$$

Since $W$ is also a neighborhood of 0 in $E_{n}$, there exists neighborhoods of $0, S$ in $X$ and $V$ in $Y$ and an integer $m \in N$ such that

$$
Q_{2} \equiv(2 S)^{\stackrel{n}{ }} \times V^{\underline{m}} \times L^{N \backslash \underline{n+m}} \subset W .
$$

Thus we have

$$
S_{-}^{n} \times L^{N \backslash \underline{n}} \subset \frac{1}{2} Q_{1}+\frac{1}{2} Q_{2} \subset W
$$

But $S^{n} \times L^{N \backslash n}$ is a neighborhood of $X^{N}$, which proves our claim.

Finally we note on the one hand that, if $B$ is the unit ball in $X$, then $B^{N}$ is bounded in $E=X^{N}$, but, on the other hand, since $B$ is not finite dimensional and so unbounded in $Y, B^{N}$ is unbounded in each $E_{n}$.

Example 2. Let $X, Y$, and $B$ be as in Example 1, $Z$ a proper dense linear subspace of $X$ endowed with its finest locally convex topology, and $D$ be $B \cap Z$. For each $n \in N$, let $E_{n}$ be the locally convex product $X^{\underline{n}} \times Y \times$ $Z^{N \backslash n+1}$ and let $E$ be the inductive limit ind $\lim E_{n}$ (with respect to the identity mapping id: $\left.E_{n} \rightarrow E_{n+1}\right)$. Since every linear subspace of $Z$ is closed, it is evident that each $E_{n}$ is closed in $E_{n+1}$ : that $\mathrm{H}-1$ holds.

Claim. $D^{N}$ is bounded in $E$. Let $G$ be any convex neighborhood of 0 in $E$. Then $G \cap E_{1}$ is a neighborhood of 0 in $E_{1}$ and so there exist neighborhoods of $0, U$ in $Y$ and $A$ in $Z$ such that

$$
Q_{1}=U_{-}^{2} \times A_{-}^{n} \times Z^{N \backslash \underline{n+2}} \subset G \text { for some } n \in N \text {. }
$$

Further $G \cap E_{n}$ is a neighborhood of 0 in $E_{n}$ and so there exist neighborhoods of $0, S$ of $X, T$ of $Y$, and $V$ of $Z$ such that 


$$
Q_{2}=(2 S) \stackrel{n+2}{\underline{n}} \times T \times V^{m} \times Z^{N \backslash \underline{n+m+3}} \subset G \quad \text { for some } m \in N .
$$

The set $S^{\underline{n+2}} \times Z^{N \backslash \underline{n+2}}$ absorbs $D^{N}$ and

$$
S \stackrel{n+2}{ } \times Z^{N \backslash \underline{n+2}} \subset \frac{1}{2} Q_{1}+\frac{1}{2} Q_{2} \subset G .
$$

Hence $G$ absorbs $D$, which proves our claim.

If $a \in B^{N} \cap E$, then $a \in B^{N} \cap E_{n}$ for some $n \in N$ and so $a$ is a limit of a sequence in $D^{N}$. Hence $B^{N} \cap E$ is the closure of the bounded set $D^{N}$, and so is bounded itself. But evidently $B^{N} \cap E$ is not contained in any $E_{n}$. Thus, though H-1 holds, it follows from the theorem of this paper that $\mathrm{H}-3$ does not.

\section{REFERENCES}

1. J. Dieudonné and L. Schwartz, La dualité dans les espaces (F) et (EF), Ann. Inst. Fourier Grenoble 1 (1949), 61-101.

2. J. Horváth, Topological vector spaces and distributions, Vol. I, Addison-Wesley, Reading, Mass., 1966.

3. J. L. Kelley and I. Namioka, Linear topological spaces, Van Nostrand, Princeton, N.J., 1963.

Department of Mathematics, Washington State Universtry, Pulluman, Washington 99163 